\title{
Effects of exercise using a mobile device on cardiopulmonary function, metabolic risk factors, and self-efficacy in obese women
}

\author{
Dae-Young Kim* \\ Department of Sports Healthcare, College of Humanities \& Social Sciences, Inje University, Gimhae, Korea
}

The purpose of this study was to determine the effects of a 12-week walking exercise using a mobile device on cardiopulmonary function, metabolic risk factors, and self-efficacy in obese middle-aged women. For this study, 14 middle-aged subjects with obesity were included and performed walking exercise of 50\%-60\% maximum oxygen uptake intensity 3 times a week for 12 weeks. As a result, weight, \% body fat, body mass index, and waist circumference significantly reduced. In addition, systolic blood pressure, diastolic blood pressure, mean arterial pressure, and hemoglobin $A_{1 c}$ significantly reduced, while high-density lipoprotein cholesterol and flexibility significantly increased. Furthermore, exercise using a mobile device significantly increased self-efficacy. In conclusion, walking exercise using a mobile device is a good method to constantly increase the exercise participation rate, as it improves metabolic risk factor and obesity index, and increases self-efficacy.

Keywords: Mobile device, Exercise, Metabolic risk factor, Self-efficacy, Obese women

\section{INTRODUCTION}

Obesity is a state of excessive accumulation of body fat surpassing the normal range that has a negative effect on the body. The incidence of obesity is increasing owing to the lack of activity in comparison with calorie intake, absence of balanced meals, and sedentary lifestyle. In Korea, the adult population with a body mass index (BMI) of $>25 \mathrm{~kg} / \mathrm{m}^{2}$ has continuously increased from 2005 to 2013 (Ministry for Health and Welfare, 2017). Obesity induces various mechanisms that lead to many diseases and increases the incidence of diseases such as metabolic syndrome, diabetes, and dyslipidemia. Among these diseases, metabolic syndrome has $\geq 3$ of the 5 risk factors, which are waist circumference (WC), glucose, high-density lipoprotein cholesterol (HDLC) level, triglyceride (TG) level, and blood pressure (BP). In other words, metabolic syndrome is a collection of risk factors associated with the increased prevalence of cardiovascular diseases, diabetes, and stroke. Lack of exercise is reported as the biggest reason for the development of metabolic syndrome, which negatively correlates with physical activity (Farrell et al., 2004; Roberts et al., 2013). In addition, the prevalence of metabolic syndrome increases with age and is more prevalent in men than in women (Lee and Kim, 2016). Thus, as obesity and metabolic syndrome lead to increase in the prevalence of various chronic diseases, the need for regular exercise is greatly emphasized.

Through many studies, regular physical activity is known to prevent obesity, and physical activity of 1,000 kcal per week or increase of $1 \mathrm{MET}$ (metabolic equivalent task) in cardiorespiratory fitness is known to reduce mortality by approximately 20\% (Myers et al., 2003). In addition, weight loss through increased physical activity is a highly effective method for prevention and improvement of chronic diseases. However, although the effects and importance of regular exercise are emphasized, the actual amount of activity remains insufficient.

Many studies have been conducted to promote physical activity. Recently, data on the amount of energy consumption and physical

\footnotetext{
${ }^{*}$ Corresponding author: Dae-Young Kim (D) https://orcid.org/0000-0002-4662-4463 Department of Sports Health Care, College of Humanities \& Social Sciences, Inje University, 197 Inje-ro, Gimhae 50834, Korea

Tel: +82- 55-320-3171, Fax: +82-55-320-3545, E-mail: cdps21@inje.ac.kr Received: August 20, 2018 / Accepted: September 17, 2018
}

This is an Open Access article distributed under the terms of the Creative Commons Attribution Non-Commercial License (http://creativecommons.org/licenses/by-nc/4.0/) which permits unrestricted non-commercial use, distribution, and reproduction in any medium, provided the original work is properly cited. 
activity have become easier to collect using devices such as an accelerometer during exercise. These devices function as a motivation factor for exercising through the self-monitoring function, rather than simply measuring the amount of activity and calories. Currently, wearable devices such as wristbands and linkage with a smartphone application allow determining the amount of physical activity and further serve as a factor that promotes exercise through setting up activity goals (Thompson, 2014).

The utilization of a mobile device assists the users to regularly exercise by individually setting up their goals and constantly stimulating them. On the basis of the results of such execution of exercise, self-efficacy can be improved. Self-efficacy refers to the belief one has on one's ability to perform a specific action. In addition, it is known as a predictor for the beginning and maintenance of regular exercise (Williams et al., 2008). Such self-efficacy is affected by personal experience, indirect experience, social persuasion, and physiological factors, among which personal experience has the biggest influence. The use of a mobile device is a good way to increase self-efficacy by providing an opportunity to directly confirm one's amount of activity, not only offering motivation but also showing individual success. However, research is lacking for specifically analyzing the effects of exercise utilizing mobile devices on various metabolic risk factors and self-efficacy. Therefore, the purpose of this study was to verify the effects of walking exercise using a mobile device on cardiopulmonary function, metabolic risk factors, and self-efficacy in obese women.

\section{MATERIALS AND METHODS}

\section{Study subjects}

The subjects were 14 obese middle-aged women, between 40 and 60 years of age, who had no other medical conditions. The subjects were selected through a baseline test by examining their age, physical activity level, weight change over the past year, smoking status, and current medication. Subjects who satisfied the following criteria were selected: WC of $\geq 80 \mathrm{~cm}$, BMI of $\geq 25$ $\mathrm{kg} / \mathrm{m}^{2}$, and exercise $<2$ times a week. All the subjects received a full explanation of the content and purpose of the study, agreed to participate by providing written consent, and then participated in the study. The study subjects' characteristics are shown in Table 1 .

\section{Analysis categories and methods \\ Physique and body composition}

As body composition variables, BMI, \% body fat, and WC were measured. After height $(\mathrm{cm})$ and weight $(\mathrm{kg})$ were measured us-
Table 1. Physical characteristics of the subjects $(n=14)$

\begin{tabular}{lr}
\hline Characteristic & Mean \pm SD \\
\hline Age $(\mathrm{yr})$ & $47.93 \pm 6.38$ \\
Height $(\mathrm{cm})$ & $158.61 \pm 4.95$ \\
Weight $(\mathrm{kg})$ & $68.69 \pm 6.31$ \\
Body fat $(\%)$ & $38.77 \pm 3.53$ \\
Body mass index $\left(\mathrm{kg} / \mathrm{m}^{2}\right)$ & $27.34 \pm 2.36$ \\
\hline
\end{tabular}

$\mathrm{SD}$, standard deviation.

ing an automated measuring instrument BSM330 (InBody, Seoul, Korea), BMI was calculated using the equation weight $(\mathrm{kg}) /$ height $\left(\mathrm{m}^{2}\right)$. For $\%$ body fat, the body composition analyzer (Inbody770, InBody) was used. WC was measured using a tape measure up to the tenth place.

\section{Blood test}

For all the subjects, a 12-hr fasting blood test was conducted. Blood samples were collected at the same time and condition before and after exercise (12 weeks after exercise). For the blood test, Samsung LABGEO PT10 (Samsung Electronics, Suwon, Korea) was used to perform a clinical chemistry analysis for whole blood samples. Blood glucose level, atherosclerosis index (AI), total cholesterol (TC) level, TG level, and HDLC level were checked by injecting $70 \mu \mathrm{L}$ of the sample into the cartridge.

\section{Blood pressure}

$\mathrm{BP}$ was measured in the right arm using an automatic BP monitor (BPBIO320, InBody) after taking a rest for $10 \mathrm{~min}$ in a sitting position. The measurement was performed twice, and the mean value was used for data processing. The time interval between measurements was $5 \mathrm{~min}$. The mean arterial pressure (MAP) was computed as MAP = diastolic BP (DBP) $+([$ systolic BP (SBP)-DBP]/3).

\section{Cardiopulmonary function test}

For cardiopulmonary function, the subjects maintained fasting for $4 \mathrm{hr}$, and a treadmill and gas analyzer were used for measurement. By using the modified Bruce protocol, a treadmill maximum oxygen uptake $\left(\mathrm{VO}_{2 \max }\right)$ test was performed. The maximum capacity criteria were a respiratory exchange ratio of $\geq 1.15$, rating of perceived exertion of $\geq 17, \mathrm{VO}_{2}$ value not increasing even when the exercise intensity was increased, and subjects asking to stop the test. Oxygen consumption and heart rate were measured using a treadmill and metabolic gas analyzer system (Quark B2, Cosmed, Rome, Italy). Then, individual regression equation between oxy- 
gen consumption and heart rate per minute was computed, and individual heart rate per minute, which is $50 \%-60 \%$ of the maximum oxygen consumption, was computed.

\section{Exercise program using a mobile device}

The aerobic exercise program used in this study was conducted as a 12-week walking exercise performed 3 times per week at the exercise intensity corresponding to $50 \%-60 \%$ of the maximum oxygen consumption. To determine the target calorie consumption, the energy consumption was calculated using the automatic heart rate monitor (Polar Heart Monitor, Polar M400, Polar Electro, Kempele, Finland) to check the status of the target heart rate set during the walking exercise program. To set the energy consumption through each exercise session identical to $400 \mathrm{kcal}$ per session and 1,200 kcal per week (exercise 3 times a week), the duration of exercise was set differently between 60 and $80 \mathrm{~min}$, depending on the individual's capacity. On the basis of the maximum oxygen consumption obtained from the Bruce protocol, the subjects were classified into 4 groups. Each group participated in the exercises using a mobile application under the guidance of an experienced exercise specialist. The exercise specialists educated the subjects on how to use the application, how to check during exercise, and how to search the results after exercise.

\section{Self-efficacy and health-related physical fitness}

For the self-efficacy measurement tool, the self-efficacy scale developed by Sherer et al. (1982) was used. The self-efficacy questionnaire consists of two questions on the beginning of action, six questions on effort, five questions on the willingness to continue despite adversity, two questions on achievement, and two questions on confidence. It consists of 17 questions with a 5-point Likert scale. A high score indicates high self-efficacy.

Health-related physical fitness includes muscle strength, muscle endurance, flexibility, cardiopulmonary endurance, and body composition. This study used a physical fitness tester (Helmas III,

Table 2. Changes in the obesity indexes and cardiopulmonary function after 12 weeks of exercise training

\begin{tabular}{lcccc}
\hline Variable & Pre & Post & $\Delta$ score & $P$-value \\
\hline Weight $(\mathrm{kg})$ & $68.69 \pm 6.31$ & $67.22 \pm 6.42$ & $-1.46 \pm 1.64$ & 0.005 \\
Body fat $(\%)$ & $38.77 \pm 3.53$ & $37.76 \pm 3.62$ & $-1.02 \pm 1.62$ & 0.034 \\
$\mathrm{BMl}\left(\mathrm{kg} / \mathrm{m}^{2}\right)$ & $27.34 \pm 2.36$ & $26.72 \pm 2.42$ & $-0.61 \pm 0.67$ & 0.005 \\
$\mathrm{VO}_{2 \max }(\mathrm{mL} / \mathrm{kg} / \mathrm{min})$ & $26.03 \pm 5.71$ & $27.64 \pm 5.25$ & $1.60 \pm 3.10$ & 0.075
\end{tabular}

Values are presented as mean \pm standard deviation.

$\Delta$ score, change in the score from before the exercise program to 12 weeks after; $\mathrm{BMI}$, body mass index; $\mathrm{VO}_{2 \max }$, maximum oxygen uptake.
$\mathrm{O}_{2}$ Run, Seoul, Korea) to measure muscle strength (lower extremities), muscle endurance (push-ups), flexibility (sitting and bending forward), and cardiopulmonary endurance (graded exercise test).

\section{Data processing}

In this study, the mean and standard deviation of measurements were obtained using the IBM SPSS Statistics ver. 23.0 (IBM Co., Armonk, NY, USA). Pre- and postchange values $(\Delta)$ were computed, and the paired samples $t$-test was performed to compare before and after 12 weeks of exercise. The significance level was set at $P$-value of $<0.05$.

\section{RESULTS}

\section{Change in anthropometric measurements and cardiopulmonary function}

Compared with before exercise, weight $(P=0.005), \%$ body fat $(P=0.034)$, and BMI $(P=0.005)$ were significantly reduced, but no significant differences in $\mathrm{VO}_{2 \max }$ and the cardiopulmonary function index were observed (Table 2).

\section{Change in metabolic risk factors}

Compared with before exercise, SBP $(P=0.003)$, DBP $(P=$ $0.048)$, MAP $(P=0.011)$, glycosylated hemoglobin $\left(\mathrm{HbA}_{1 \mathrm{c}}\right)$ level $(P=0.006)$, and WC $(P=0.024)$ were significantly reduced, while HDLC level $(P=0.037)$ was significantly increased. No signifi-

Table 3. Changes in the metabolic risk factors after 12 weeks of exercise training

\begin{tabular}{lcccc}
\hline Variable & Pre & Post & $\Delta$ score & $P$-value \\
\hline TG $(\mathrm{mg} / \mathrm{dL})$ & $131.64 \pm 46.08$ & $124.79 \pm 46.92$ & $-6.86 \pm 50.29$ & 0.618 \\
TC $(\mathrm{mg} / \mathrm{dL})$ & $217.86 \pm 37.92$ & $216.36 \pm 41.49$ & $-1.50 \pm 28.83$ & 0.849 \\
HDLC $(\mathrm{mg} / \mathrm{dL})$ & $54.79 \pm 11.05$ & $57.79 \pm 10.86$ & $3.00 \pm 4.82$ & 0.037 \\
Al & $3.05 \pm 0.67$ & $2.85 \pm 0.96$ & $-0.20 \pm 0.57$ & 0.219 \\
Glucose & $106.36 \pm 28.77$ & $95.93 \pm 12.62$ & $-10.42 \pm 18.86$ & 0.059 \\
HbA $_{1 c}$ & $6.12 \pm 0.56$ & $5.82 \pm 0.36$ & $-0.31 \pm 0.35$ & 0.006 \\
SBP $(\mathrm{mmHg})$ & $129.21 \pm 13.77$ & $116.36 \pm 13.14$ & $-12.86 \pm 13.09$ & 0.003 \\
DBP $(\mathrm{mmHg})$ & $86.00 \pm 16.87$ & $78.50 \pm 11.69$ & $-7.50 \pm 12.84$ & 0.048 \\
MAP $(\mathrm{mmHg})$ & $100.40 \pm 15.41$ & $91.12 \pm 11.15$ & $-9.29 \pm 11.70$ & 0.011 \\
WC $(\mathrm{cm})$ & $91.92 \pm 5.29$ & $90.04 \pm 6.01$ & $-1.89 \pm 2.75$ & 0.024 \\
\hline
\end{tabular}

Values are presented as mean \pm standard deviation.

$\Delta$ score, changes in the score from before the exercise program to 12 weeks after; TG, triglycerides; TC, total cholesterol; HDLC, high-density lipoprotein cholesterol; $\mathrm{Al}$, atherosclerosis index; $\mathrm{HbA}_{1 \mathrm{c}}$ glycosylated hemoglobin; SBP, systolic blood pressure; DBP, diastolic blood pressure; MAP, mean arterial pressure; WC, waist circumference. 
Table 4. Changes in health-related physical fitness and self-efficacy

\begin{tabular}{lcccc}
\hline Variable & Pre & \multicolumn{1}{c}{ Post } & \multicolumn{1}{c}{$\Delta$ score } & $P$-value \\
\hline Muscle strength & $37.91 \pm 14.86$ & $36.12 \pm 12.77$ & $-1.78 \pm 5.65$ & 0.259 \\
Muscle endurance & $22.36 \pm 16.90$ & $27.14 \pm 14.88$ & $4.79 \pm 10.19$ & 0.102 \\
Flexibility & $11.77 \pm 7.00$ & $14.11 \pm 5.33$ & $2.34 \pm 3.83$ & 0.040 \\
Self-efficacy & $59.14 \pm 9.65$ & $62.79 \pm 9.94$ & $3.64 \pm 6.28$ & 0.049
\end{tabular}

Values are presented as mean \pm standard deviation.

$\Delta$ score, change in the score from before the exercise program to 12 weeks after.

cant differences in TG level, TC level, AI, and glucose level were observed (Table 3).

\section{Changes in health related physical fitness and self-efficacy}

Compared with before exercise, a significant difference was observed only in flexibility $(P=0.040)$, which is a health-related physical fitness factor. No significant differences were found in muscle strength and endurance. Self-efficacy $(P=0.049)$ significantly increased as compared with before exercise (Table 4).

\section{DISCUSSION}

This study analyzed the effects of a 12-week walking program using a mobile device on changes in cardiopulmonary function, metabolic risk factors, and self-efficacy in obese women. Weight, $\%$ body fat, and BMI were significantly reduced, but no significant difference in $\mathrm{VO}_{2 \max }$, a cardiopulmonary index, was observed. This aligns with the study results of a study conducted by Kim (2017) that showed a reduction in weight, BMI, and \% body fat in obese middle-aged women after 12 weeks of walking exercise. In this study, cardiorespiratory fitness index did not show a significant increase. This contradicts with the results of previous studies that reported an increase in cardiorespiratory fitness through exercise (Kim et al., 2012; Thompson et al., 2003). In this study, the improvement in cardiorespiratory fitness showed no significant difference because the walking exercise was performed at a relatively low intensity.

In this study, regular exercise significantly decreased SBP, DBP, MAP, $\mathrm{HbA}_{\mathrm{lc}}$ level, and WC among the metabolic risk factors in obese women, while HDLC level was significantly increased. Owing to the significant decrease in SBP and DBP, MAP also significantly decreased. In many previous studies that analyzed the relationship between exercise and BP, regular exercise helped the management of appropriate $\mathrm{BP}$, which improved other chronic disease factors (Ochi et al., 2010; Thompson et al., 2003).

Particularly among metabolic risk factors, higher WC has a high possibility of advancing to chronic diseases such as hypertension, stroke, diabetes, and cardiovascular disease, and it is also directly associated with mortality (Kim et al., 2009). Aside from energy intake, physical activity can reduce subcutaneous and visceral fat in the abdomen. In this study, WC was significantly reduced, which seems to be a positive effect of exercise. In addition, HDLC significantly increased. Among the metabolic risk factors, HDLC is known as a factor that reduces the risk of coronary sclerosis. Reduced body fat leads to increase in HDLC level, and the two indexes are known to be significantly correlated (LeMura and Maziekas, 2002).

In this study, fasting blood glucose levels before and after exercise did not show a significant difference, but $\mathrm{HbA}_{\mathrm{lc}}$ level was significantly reduced. $\mathrm{HbA}_{\mathrm{lc}}$ level refers to the amount of glucose bound to the hemoglobin, which is an index that shows the mean blood glucose concentration in the past 2-3 months. Regular exercise in this study seemed to be effective because it inhibited endogenous glucose production in the liver and helped control blood glucose through increased glucose uptake in the muscle (Dasgupta et al., 2007). In summary, the results of this study are consistent with those of previous studies that regular exercise reduces risk factors of obesity and metabolic syndrome, such as obesity index, BP improvement, HDLC increase, and blood glucose control (Laaksonen et al., 2002; Thompson et al., 2003).

Regular walking exercise showed a significant increase in flexibility among health-related fitness factors, but no significant differences in muscle strength and muscle endurance. Upon comparing the results of previous studies, Shin and Kim (2016) reported that 8 weeks of walking had a positive effect on muscle endurance and flexibility in obese middle-aged women. Hyun (2012) reported that 12 weeks of walking exercise improved cardiopulmonary endurance and flexibility in obese women. In this study, stretching during warm-up and cool-down exercises helped improve flexibility. However, as the exercise intervention method was walking, it does not seem to have helped much in improving muscle strength and muscle endurance.

According to this study, 12 weeks of the walking exercise program using a mobile device had a positive effect on self-efficacy. An individual with high self-efficacy has a sense of self-control that reduces negative emotions such as stress and anxiety and leads to active participation in exercise (Huh and Lim, 2009). Jo et al. (2018) reported a significant increase in self-efficacy through a 12-week exercise in adults with intellectual disabilities, and Kim et al. (2009) reported that commitment to exercise has an effect on self-efficacy. Hence, self-efficacy increases the most with 
experience of success from regular exercise. The most convenient tool to confirm one's success is a mobile device. Continuously checking the user's amount of activity by using activity- and smartphone-based applications can be considered as a measure of confirming the effectiveness of lifestyle and health care. Jee (2017) reported applications to be effective in promoting motivation, and Kim et al. (2018) reported that when using activity measurement and smart-based applications to maintain regular physical activity for 23 weeks, $90.65 \%$ of males and $91.37 \%$ of females showed continuity. The use of mobile devices has a positive effect on self-efficacy by increasing the practice rate of exercising, which is considered to be the factor that can promote continuous participation in exercise.

In this study, 12 weeks of walking exercise was performed by obese women using a mobile device, from which positive results of reduced risk factors of obesity and metabolic syndrome were obtained, such as improvement in obesity indexes, BP, glucose control, and HDLC level. In addition, by using mobile devices, self-efficacy is expected to increase through self-monitoring by visually checking one's level of activity, which would lead to increased practice rate of exercising. Currently, studies that use mobile devices are lacking. Further studies will be needed to determine the effect of various devices on exercise.

\section{CONFLICT OF INTEREST}

No potential conflict of interest relevant to this article was reported.

\section{ACKNOWLEDGMENTS}

This work was supported by the 2017 Inje University Research Grant.

\section{REFERENCES}

Dasgupta K, Chan C, Da Costa D, Pilote L, De Civita M, Ross N, Strachan I, Sigal R, Joseph L. Walking behaviour and glycemic control in type 2 diabetes: seasonal and gender differences-Study design and methods. Cardiovasc Diabetol 2007;6:1.

Farrell SW, Cheng YJ, Blair SN. Prevalence of the metabolic syndrome across cardiorespiratory fitness levels in women. Obes Res 2004;12: 824-830.

Huh JY, Lim HM. The relationship between self-efficacy and exercise adherence in behavioral activation/behavioral inhibition system. J Sport
Leis Stud 2009;35:1039-1050.

Hyun SH. Effect of 12 weeks walking exercise for physical fitness, body composition, and blood lipids in obese women. J Korean Assoc Secur Saf 2012;8:135-150.

Jee H. Review of researches on smartphone applications for physical activity promotion in healthy adults. J Exerc Rehabil 2017;13:3-11.

Jo G, Rossow-Kimball B, Lee Y. Effects of 12-week combined exercise program on self-efficacy, physical activity level, and health related physical fitness of adults with intellectual disability. J Exerc Rehabil 2018;14: 175-182.

Kim DJ, Kim MS, Park NY, Lee SG, Lee YK. Effect of activity tracker \& application use on adherence, metabolic syndrome factor and body composition in middle and senior-aged. Asian J Kinesiol 2018;20:2837.

Kim DJ, Kwon CK, Choi DJ, Ka KH, Kim TM, Kim BT, Lee BK, Hwang JH, Ann ES, Kim DY. Effects of nutritional education and exercise intervention on improvement of diet intakes and metabolic risk factors in obese middle aged women. Korean J Exerc Nutr 2009;13:179-184.

Kim MS, Kim SH, Lee, SH. Effects of regular walking exercise on cardiorespiratory fitness, metabolic syndrome risk factors in middle-aged women. J Korean Soc Living Environ Sys 2012;19:371-380.

Kim SH. Effects of a 12-week Walking exercise on body composition, metabolic-related variables and stress-related variables in middle-age obese women. Korea J Sports Sci 2017;26:867-877.

Laaksonen DE, Lakka HM, Niskanen LK, Kaplan GA, Salonen JT, Lakka TA. Metabolic syndrome and development of diabetes mellitus: application and validation of recently suggested definitions of the metabolic syndrome in a prospective cohort study. Am J Epidemiol 2002;156: 1070-1077.

Lee H, Kim BH. Physical activity disparities by socioeconomic status among metabolic syndrome patients: The Fifth Korea National Health and Nutrition Examination Survey. J Exerc Rehabil 2016;12:10-14.

LeMura LM, Maziekas MT. Factors that alter body fat, body mass, and fat-free mass in pediatric obesity. Med Sci Sports Exerc 2002;34:487496.

Ministry for Health and Welfare. 2008-2016 Local health statistics. Sejong (Korea): Ministry for Health and Welfare; 2017.

Myers J, Gullestad L, Bellin D, Ross H, Vagelos R, Fowler M. Physical activity patterns and exercise performance in cardiac transplant recipients. J Cardiopulm Rehabil 2003;23:100-106.

Ochi M, Kohara K, Tabara Y, Kido T, Uetani E, Ochi N, Igase M, Miki T. Arterial stiffness is associated with low thigh muscle mass in middle-aged to elderly men. Atherosclerosis 2010;212:327-332.

Roberts CK, Hevener AL, Barnard RJ. Metabolic syndrome and insulin resistance: underlying causes and modification by exercise training. 
Compr Physiol 2013;3:1-58.

Sherer M, Maddux JE, Mercandante B, Prentice-Dunn S, Jacobs B, Rogers RW. The self-efficacy scale: construction and validation. Psychol Rep 1982;51:663-671.

Shin JH, Kim MS. The effects on body composition, health related physical fitness and metabolic syndrome factors of working exercise program in obese middle-aged women. J Coach Dev 2016;18:39-46.

Thompson PD, Buchner D, Pina IL, Balady GJ, Williams MA, Marcus BH, Berra K, Blair SN, Costa F, Franklin B, Fletcher GF, Gordon NF, Pate RR, Rodriguez BL, Yancey AK, Wenger NK; American Heart Association Council on Clinical Cardiology Subcommittee on Exercise, Rehabilitation, and Prevention; American Heart Association Council on Nutrition, Physical Activity, and Metabolism Subcommittee on Physi- cal Activity. Exercise and physical activity in the prevention and treatment of atherosclerotic cardiovascular disease: a statement from the Council on Clinical Cardiology (Subcommittee on Exercise, Rehabilitation, and Prevention) and the Council on Nutrition, Physical Activity, and Metabolism (Subcommittee on Physical Activity). Circulation 2003;107:3109-3116.

Thompson WR. Worldwide survey of fitness trends for 2015: what's driving the market. ACSM's Health Fit J 2014;18:8-17.

Williams DM, Lewis BA, Dunsiger S, Whiteley JA, Papandonatos GD, Napolitano MA, Bock BC, Ciccolo JT, Marcus BH. Comparing psychosocial predictors of physical activity adoption and maintenance. Ann Behav Med 2008;36:186-194. 